\title{
Damping of helioseismic modes in steady state
}

\author{
B. Pintér ${ }^{1}$, R. Erdélyi ${ }^{2}$, and R. New ${ }^{1}$ \\ 1 School of Science and Mathematics, Hallam University, Howard Street, Sheffield S1 1WB, UK \\ e-mail: r.new@shu.ac.uk \\ 2 Space \& Atmosphere Research Center, Dept. of Applied Mathematics, University of Sheffield, Hicks Building, \\ Hounsfield Road, Sheffield S3 7RH, UK \\ e-mail: Robertus@sheffield.ac.uk
}

Received 26 March 2001 / Accepted 12 April 2001

\begin{abstract}
The effects of an equilibrium flow in the internal regions of the Sun are studied on the damping of helioseismic $f$ - and $p$-modes. The Sun is modeled as a multi-layered plasma, where the upper parts, representing the chromosphere and corona, are embedded in a horizontally unidirectional though vertically inhomogeneous magnetic field, while the lower part, representing the sub-photospheric polytropic region, is in a steady equilibrium state. The steady state sub-surface region can be considered as a first approximation of dynamic motions (e.g., differential rotation, sub-surface flows, meridional flows, convective motion, etc.).

The frequencies and the line-widths of eigenmodes are affected by sub-surface flow and atmospheric magnetic fields. A key contribution to the effects comes from the universal mechanism of resonant absorption.

When both atmospheric magnetic field and sub-surface flows are present, a complex picture of competition between these two effects is found. The theoretically predicted frequency shifts in a steady state show promise of explaining the observed effects. Changes in damping of $f$ - and $p$-modes caused by changes (e.g. cyclic, if any) of steady state flows are predicted.
\end{abstract}

Key words. Sun: helioseismology - Sun: oscillations - Sun: interior - Sun: atmosphere - Sun: magnetic fields Sun: rotation

\section{Introduction}

Variations with solar activity of the characteristic parameters of solar acoustic vibrations ( $p$-modes) have been tracked over two decades with a much increased range of independent observations in the current activity cycle. Changes in frequency of up to about $0.4 \mu \mathrm{Hz}$ over the solar cycle were established in the early 1990's (Elsworth et al. 1990; Libbrecht \& Woodard 1990; Anguera Gubau et al. 1992; Rhodes et al. 1993; Elsworth et al. 1994), and detailed studies have been made possible by the high quality data sets now available from the ground-based GONG and BiSON networks and instruments such as MDI on the SOHO spacecraft (e.g. Chaplin et al. 1998; Dziembowski et al. 1998; Bhatnagar et al. 1999; Howe et al. 1999, 2000a,b; Antia \& Basu 2000). The correlations between solar activity and mode amplitudes and widths are intrinsically more difficult to study, but a consensus between observers now appears to have been reached (Chaplin et al. 2000; Komm et al. 2000a, and references therein). Modes with angular degree, $l$, up to 150 in the frequency range

Send offprint requests to: B. Pintér, e-mail: b.pinter@shu.ac.uk
1700 to $4100 \mathrm{mHz}$ show lower amplitudes and greater widths at solar maximum compared with solar minimum. Both effects are of the order of $10 \%$, with the detailed values depending somewhat upon the modes studied. Komm et al. (2000a) report that the percentage variations are found to be largely independent of $l$. However, since the absolute widths are themselves $l$-dependent (scaling approximately as inverse mode-mass across most of the spectrum, Komm et al. 2000b), the width variations are $l$ dependent. A $10 \%$ variation corresponds to an absolute width change in the approximate range 0.1 to $1 \mu \mathrm{Hz}$, depending on the mode studied.

Helioseismic techniques can also be used to study large-scale flows on the Sun, such as meridional circulation and torsional oscillations. Meridional flows with velocities in the range $10-20 \mathrm{~m} \mathrm{~s}^{-1}$ have been detected applying time-distance helioseismic techniques to MDI data obtained in 1996 (Giles et al. 1997), and there are some indications in the literature that such flows may be slower at sunspot maximum (Komm et al. 1993). Torsional oscillations were first reported in the early 1980's (Howard \& LaBonte 1980), and manifest themselves as bands in the rotation profile, the relative speed of adjacent bands being 
of the order of $5 \mathrm{~m} \mathrm{~s}^{-1}$. GONG (Howe et al. 2000c) and MDI (Toomre et al. 2000) observations over recent years show a solar cycle variation in these oscillations, with the bands drifting towards the equator during the rising phase of the Sun's current activity cycle.

The theoretical interpretation of the shifts of $f$-and $p$-modes has attracted a considerable effort in static models (e.g., Campbell \& Roberts 1989; Evans \& Roberts 1992; Jain \& Roberts 1993, 1994), while Murawski \& Roberts (1993) studied random velocity field corrections of the $f$-mode.

In this paper we describe models and present results which show the influence on the frequencies and widths of global $p$-modes of resonant coupling with MHD waves in the chromosphere. We build on earlier work (Pintér \& Goossens 1999; Pintér et al. 2001), which demonstrated the potential for this effect to cause frequency shifts and damping for a range of magnetic field geometries, by incorporating the effects of a large-scale flow on this system. The results demonstrate the principle that resonant coupling with MHD waves in a steady state might be in part responsible for the solar cycle variation of $p$-mode parameters. We indicate the direction of future work which may allow a detailed comparison.

\section{Equilibrium model and dispersion relation}

The Sun is modeled with a three-layer structure (see Fig. 1), representing the solar interior, chromosphere and corona in a Cartesian geometry. (This means our analysis is valid strictly speaking only for high degree modes, for which the horizontal wavelength is small compared to the solar radius, i.e., $l \geq 6$.)

For the solar interior we consider an adiabatic temperature profile, where the temperature increases linearly with depth with a gradient of $(\gamma-1) g$. The solar interior is in a steady state, i.e. there is a homogeneous constant equilibrium flow, $\boldsymbol{v}_{0}(\boldsymbol{z})=\left(v_{0}(z), 0,0\right)$ in the horizontal direction ( $x$-axis), where

$v_{0}(z)= \begin{cases}v, & z \geq 0 \\ 0, & z<0\end{cases}$

The solar chromosphere and corona are embedded in a unidirectional horizontal magnetic field $\boldsymbol{B}_{0}(\boldsymbol{z})=$ $\left(B_{0}(z), 0,0\right)$. The chromosphere is a transitional layer, where the strength of the magnetic field increases continuously from zero, giving rise to slow and Alfvén continua in the frequency spectrum of the multi-layered atmosphere. The magnetic field strength decreases exponentially with height in the corona resulting in a constant coronal Alfvén speed of this model (see Fig. 1). The choice of a discontinuous equilibrium flow may cause Kelvin-Helmholtz instability, however the characteristic wave number, $k^{-1} \geq L$, which means this effect is negligible.

Let us introduce the Eulerian perturbation of the total pressure (as the sum of the thermal plasma pressure and the magnetic pressure), $P \equiv p+\boldsymbol{B}_{0} \cdot \boldsymbol{B} / \mu$, and the vertical component of the Lagrangian displacement, $\xi_{z}$.

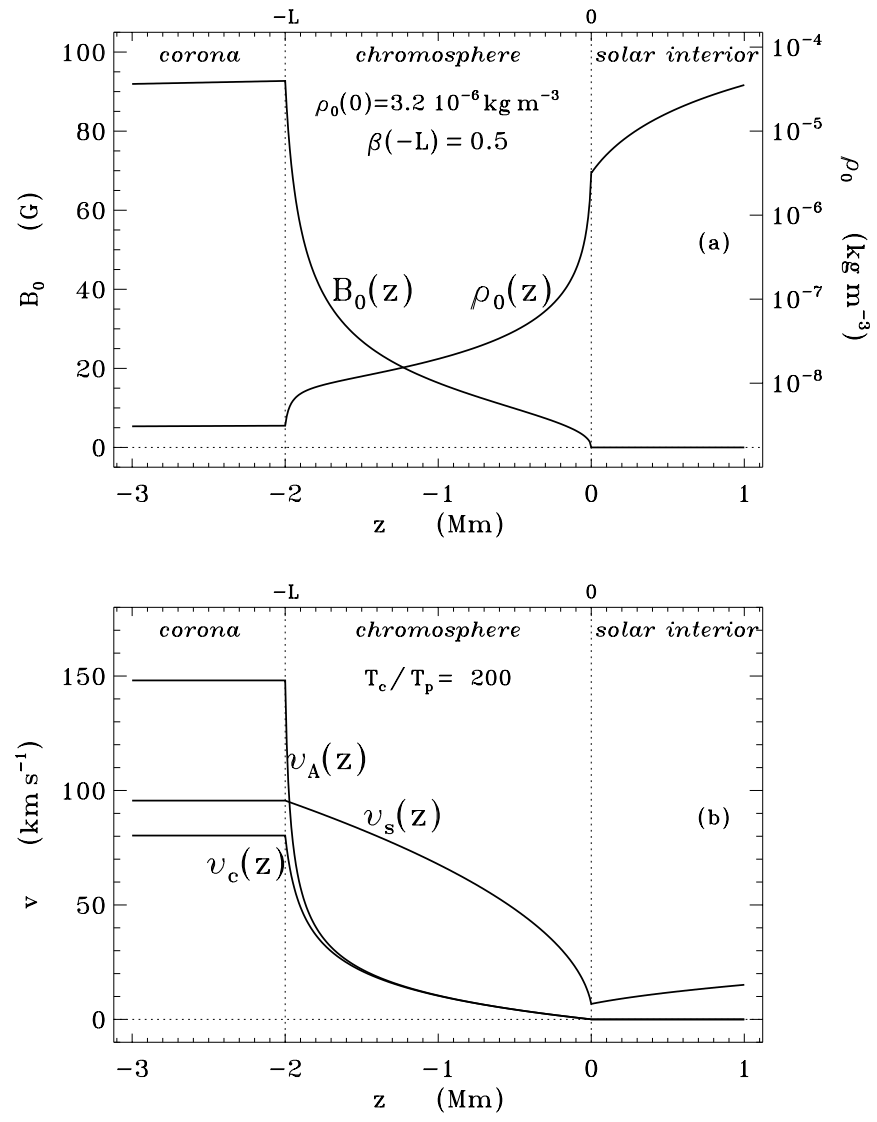

Fig. 1. a) The atmospheric magnetic induction, the plasma density and $\mathbf{b})$ the slow $\left(v_{\mathrm{c}}\right)$, sound $\left(v_{\mathrm{s}}\right)$ and Alfvén $\left(v_{\mathrm{A}}\right)$ speeds, respectively, as function of height. The magnetic field strength increases from zero in a characteristic transitional layer (between 0 to $-L$, where $L=2 \mathrm{Mm}$ ), the characteristic velocities are constant in the corona. The solar interior is field-free and polytropic.

The linearized MHD equations can be reduced to two ODEs for $\xi_{z}$ and $P$ in Fourier space:

$D \frac{\mathrm{d} \xi_{z}}{\mathrm{~d} z}=C_{1} \xi_{z}-C_{2} \frac{\Omega}{\omega} P, \quad D \frac{\Omega}{\omega} \frac{\mathrm{d} P}{\mathrm{~d} z}=C_{3} \xi_{z}-C_{1} \frac{\Omega}{\omega} P$.

The coefficient functions $D, C_{1}, C_{2}$ and $C_{3}$ are

$D(z)=\rho_{0}\left(v_{\mathrm{s}}^{2}+v_{\mathrm{A}}^{2}\right)\left(\Omega^{2}-\omega_{\mathrm{c}}^{2}\right)\left(\Omega^{2}-\omega_{\mathrm{A}}^{2}\right)$,

$C_{1}(z)=-g \rho_{0} \Omega^{2}\left(\Omega^{2}-\omega_{\mathrm{A}}^{2}\right)$,

$C_{2}(z)=\Omega^{4}-k^{2}\left(v_{\mathrm{s}}^{2}+v_{\mathrm{A}}^{2}\right)\left(\Omega^{2}-\omega_{\mathrm{c}}^{2}\right)$,

$C_{3}(z)=\left[\rho_{0}\left(\Omega^{2}-\omega_{\mathrm{A}}^{2}\right)-g \frac{\mathrm{d} \rho_{0}}{\mathrm{~d} z}\right] D+g^{2} \rho_{0}^{2}\left(\Omega^{2}-\omega_{\mathrm{A}}^{2}\right)^{2}$,

where $\Omega(z)=\omega-k v_{0}(z)$ is the Doppler-shifted frequency of the global $f$-and $p$-modes; $\omega_{\mathrm{A}}$ and $\omega_{\mathrm{c}}$ are the local Alfvén and cusp frequencies, respectively, whose squares are given by

$\omega_{\mathrm{A}}^{2}(z)=k_{x}^{2} v_{\mathrm{A}}^{2}(z), \quad \omega_{\mathrm{c}}^{2}(z)=k_{x}^{2} v_{\mathrm{c}}^{2}(z)$.

The squares of the characteristic velocities - the sound speed $\left(v_{\mathrm{s}}\right)$, the Alfvén speed $\left(v_{\mathrm{A}}\right)$ and the cusp speed $\left(v_{\mathrm{c}}\right)$ - are given by

$v_{\mathrm{s}}^{2} \equiv \gamma p / \rho, \quad v_{\mathrm{A}}^{2} \equiv B^{2} /(\mu \rho), \quad v_{\mathrm{c}}^{2} \equiv v_{\mathrm{s}}^{2} v_{\mathrm{A}}^{2} /\left(v_{\mathrm{s}}^{2}+v_{\mathrm{A}}^{2}\right)$. 
Equations (1) govern the linear motions of a onedimensional magnetic plasma in a gravitational field. The coefficient function $D$ vanishes at $z=z_{\mathrm{c}}$ and at $z=$ $z_{\mathrm{A}}$ where the local slow or Alfvén frequency equals the Doppler shifted eigenfrequency, $\Omega$. The singularities of Eqs. (1) can be removed by including dissipation (e.g. magnetic diffusivity, resistivity) in the MHD equations (see Pintér et al. 1999). Dissipation causes damping of the eigenmodes and the related eigenfrequencies become complex. The condition for slow resonance is $\operatorname{Re}(\Omega)=\omega_{\mathrm{c}}\left(z_{\mathrm{c}}\right)$, and for Alfvén resonance it is $\operatorname{Re}(\Omega)=\omega_{\mathrm{A}}\left(z_{\mathrm{A}}\right)$. When Eqs. (1) are supplemented with boundary conditions they define an eigenvalue problem for the global $f$ - or $p$-mode frequency, $\omega$, which we solve by numerical integration for global $f$ - and $p$-modes. We study $f$ - and $p$-modes, which are trapped oscillations within the uppermost part of the convection zone, right below the photosphere $(z \geq 0)$. Hence the requirement that the energy of the global oscillations tends to zero for $z \rightarrow \pm \infty$, are suitable boundary conditions. In the special case when the horizontal component of the wave vector, $\boldsymbol{k}$, is parallel to the magnetic field lines $\left(k_{y}=0\right)$, Eqs. (1) show that the global oscillations interact resonantly only with local slow oscillations.

\section{Results and discussions}

Equilibrium profiles for the magnetic field strength, $B_{0}(z)$, density, $\rho_{0}(z)$, and characteristic sound, Alfvén and slow velocities, $v_{\mathrm{s}}(z), v_{\mathrm{A}}(z), v_{\mathrm{c}}(z)$, respectively, are assumed as shown in Fig. 1. The profile of the magnetic field is determined by taking $\beta_{\mathrm{c}} \equiv \beta(z=-2 \mathrm{Mm})=0.5$. This value represents a strong chromospheric and coronal magnetic field, which can occur in active regions of the Sun. In a non-magnetic model, $f$ - and $p$-modes can be found only with frequencies between the lower and upper cut-off frequencies, $\nu_{\mathrm{I}}$ and $\nu_{\mathrm{II}}$. Global oscillations with frequencies outside that region are leaky modes, and are not considered as eigenmodes. However, the frequency-spectrum of global oscillation modes is more plentiful in a magnetic model, as previously leaky modes couple resonantly with localized Alfvén and/or slow waves.

We present the frequencies, $\nu(\equiv \omega / 2 \pi)$, and the linewidths, $\Gamma$, of global mode frequencies as a function of the harmonic degree, $l$, for fixed values of a sub-photospheric flow, $v$.

Frequencies of the $f$ - and the first five $p$-modes are plotted with varying $l$ in Fig. 2. There are two ranges of frequencies where global mode-frequencies may occur. One is below $\nu_{\text {II }}$ and above the maximum of $\nu_{\mathrm{I}}$ and $\nu_{\mathrm{c}}$, and the other is below the minimum of $\nu_{\mathrm{I}}$ and $\nu_{\mathrm{c}}$. With increasing $l$, mode-frequencies appear slightly below $\nu_{\mathrm{II}}$ and increase until they reach $\nu_{\mathrm{I}}$. For the $f$ - and each of the $p$-modes, there is an interval of $l$, where the mode does not exist. For instance, the first $p$-mode does not exist between $l=48$ and $l=69$ (see Fig. 2). Approaching $\nu_{\mathrm{I}}$, the increase of the eigenfrequencies is faster, as the $f$ and $p$-modes transform to the Lamb-mode (also known as $a$-mode, Pintér \& Goossens 1999). In Fig. 2 the global

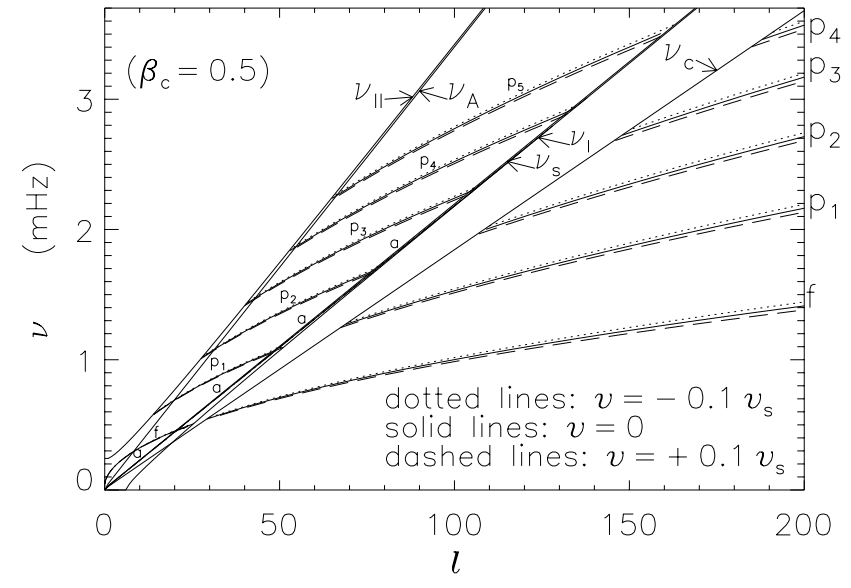

Fig. 2. Frequency spectrum of the $f$ - and the first five $p$-modes as a function of angular degree, $l$, for three different values of a sub-photospheric equilibrium plasma flow velocity, $v$.

Table 1. Slope of $\nu-v$ curves (measured in $10^{-9} \mathrm{~m}^{-1}$ ) and $\Gamma-v$ curves (measured in $10^{-12} \mathrm{~m}^{-1}$ ) for $v \in\left[-0.1 v_{\mathrm{s}}, 0.1 v_{\mathrm{s}}\right]$ for the $f$ - and $p_{1}$-modes.

\begin{tabular}{|r||r|r||r|r|}
\hline$l$ & $\mathrm{~d} \nu_{f} / \mathrm{d} v$ & $\mathrm{~d} \nu_{p 1} / \mathrm{d} v$ & $\mathrm{~d} \Gamma_{f} / \mathrm{d} v$ & $\mathrm{~d} \Gamma_{p 1} / \mathrm{d} v$ \\
\hline 20 & -4.67 & -4.68 & 0.00 & 0.0 \\
40 & -9.24 & -9.23 & 0.01 & 0.0 \\
60 & -13.78 & - & 0.3 & 0.0 \\
80 & -18.31 & -18.27 & 2.4 & 0.2 \\
100 & -22.81 & -22.75 & 16.1 & 1.1 \\
120 & -27.31 & -27.20 & 56.5 & 4.3 \\
140 & -31.83 & -31.62 & -18.7 & 15.6 \\
160 & -36.23 & -36.02 & -158.0 & 51.7 \\
180 & -40.60 & -40.39 & -178.0 & 158.5 \\
200 & -44.95 & -44.80 & -164.1 & 404.3 \\
\hline
\end{tabular}

frequencies are plotted for three different cases: a) when the sub-photospheric plasma flows anti-parallel to the magnetic field lines, b) when the plasma does not move, and c) when the flow is parallel to the magnetic field lines. The maximal velocity amplitude is one tenth of the photospheric sound speed $\left(v= \pm 0.1 v_{\mathrm{s}} \approx \pm 676 \mathrm{~m} \mathrm{~s}^{-1}\right)$. A change in the direction of such a flow would shift the frequencies of global oscillation modes by up to tens of $\mu \mathrm{Hz}$. Such a large effect would be easily detectable. There is a general tendency that an equilibrium plasma flow (anti)parallel to a horizontal magnetic field decreases (increases) the frequencies of global oscillations. The dominant effect on the frequencies is the Doppler effect: the frequencies are shifted by $-v \lambda^{-1}$, where $\lambda\left(=2 \pi(l(l+1))^{-2} R_{\odot}\right)$ is the wavelength. For flow velocities $v \in\left[-0.1 v_{\mathrm{s}}, 0.1 v_{\mathrm{s}}\right]$ the ratio of the frequency shift to the flow velocity, $(\nu(v ; l)-\nu(v=0 ; l)) v^{-1}$ is listed in Table 1 for the $f$ - and $p_{1}$-modes. The $f$-mode and the other $p$-modes show similar sensitivities to the sub-surface flow. However, for faster flows, which are not observed in the Sun, an additional $l$-dependent influence beside the Doppler shift was also found, which makes the flow-effect non-linear. Note that the frequency of the 


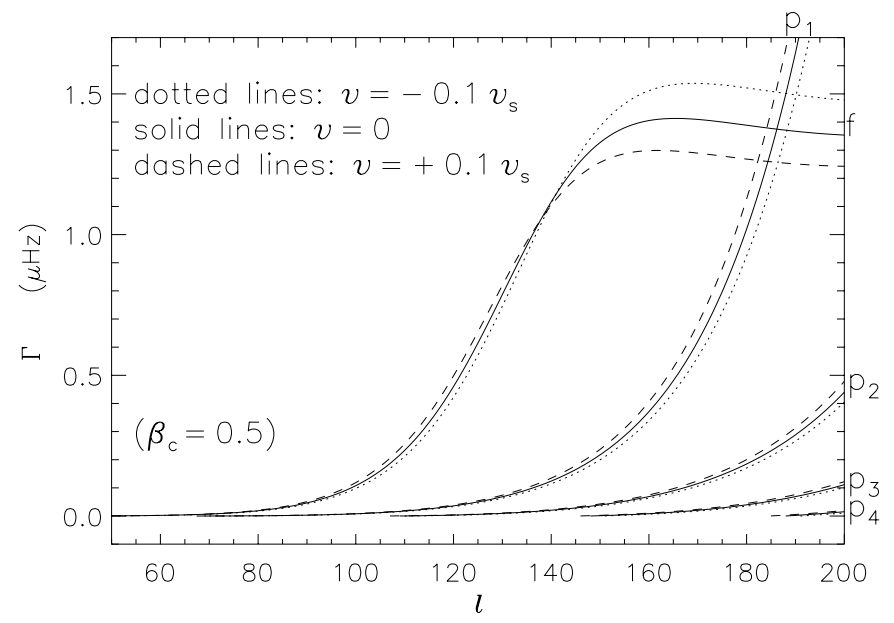

Fig. 3. Line-width of the $f$ - and the first four $p$-mode frequencies as a function of angular degree, $l$, for three different values of flow velocity, $v$.

$a$-mode is always close to $\nu_{\mathrm{s}}(l)$, hence it is not sensitive to the flow.

A global mode with frequencies falling in the slow continuum $\left(\nu<\nu_{\mathrm{c}} \equiv \nu(z=-L)\right)$ can couple resonantly to a local slow mode at a height where $\nu_{\mathrm{c}}(z)=\nu$. In the vicinity of the resonant position dissipation becomes important causing damping of such a resonant global mode. The frequencies become complex with a negative imaginary part. The greater the damping of a mode, the wider is the mode's peak in the frequency-spectrum, the linewidth being related to the imaginary part of the frequency of a damped mode by $\Gamma=-2 \operatorname{Im} \nu$. The contribution of this effect to the line-widths of the $f$ - and the first four $p$-modes is plotted in Fig. 3 as a function of $l$ for three arbitrary values of a sub-photospheric plasma-flow. Entering the slow continuum, the line-width of a mode increases from zero with increasing $l$. In most cases a horizontal plasma flow parallel to the atmospheric magnetic field lines results in a narrower line-width, while plasma flowing in the anti-parallel direction causes wider line-widths. The line-widths can vary by some tenths of $\mu \mathrm{Hz}$ when the equilibrium flow, representing some large-scale sub-surface motions, changes from $v=-0.1 v_{\mathrm{s}}$ to $v=0.1 v_{\mathrm{s}}$. This effect contributes to the line-widths only above the threshold of $l$ for which $\nu<\nu_{\mathrm{c}}$. For example, the thresholds for the $f$-, $p_{1^{-}}, p_{2^{-}}, p_{3^{-}}$and $p_{4^{-}}$modes are $l=29,69,109,148,188$, respectively.

Table 1 also shows the sensitivity of line-width of the $f$ - and $p_{1}$-modes to an equilibrium flow varying between $v \in\left[-0.1 v_{\mathrm{s}}, 0.1 v_{\mathrm{s}}\right]$. The ratio $(\Gamma(v ; l)-\Gamma(v=0 ; l)) v^{-1}$ is given for different values of $l$. The line-width of the $f$-mode increases or decreases linearly with $v$ in the interval $v \in$ $\left[-0.1 v_{\mathrm{s}}, 0.1 v_{\mathrm{s}}\right]$, while that of the $p$-mode always increases, also linearly in the given interval of $v$ and $l$. For larger $l$ the effects are more complicated, and the line-width of $p$-modes can also decrease with $v$. In addition, for larger values of $v$ the dependence becomes non-linear.
After an investigation of this simple model of a subphotosperic flow and atmospheric magnetic effects on shifts and line-widths of $f$ - and $p$-mode frequencies within the limits of the present model we expect more realistic results in a future solar model. Considering, for example, spherical geometry would make the models sensitive to splitting such as in Roth \& Stix (1999), and we will also incorporate a more realistic treatment of the magnetic field.

Acknowledgements. The authors thank B. Roberts, M. S. Ruderman, P. M. Giles, Y. Taroyan and M. Goossens for discussions and comments. RE acknowledges M. Kéray for patient encouragement. BP is grateful to PPARC for financial support. BP \& RE also acknowledge the financial support obtained from the NSF, Hungary (OTKA, Ref. No. TO32462).

\section{References}

Antia, H. M., \& Basu, S. 2000, ApJ, 541, 442

Anguera Gubau, M., Palle, P. L., Perez Hernandez, F., et al. 1992, A\&A, 255, 363

Bhatnagar, A., Jain, K., \& Tripathy, S. C. 1999, ApJ, 521, 885

Chaplin, W. J., Elsworth, Y., Isaak, G. R., et al. 1998, MNRAS, 300, 1077

Chaplin, W. J., Elsworth, Y., Isaak, G. R., et al. 2000, MNRAS, 313, 32

Dziembowski, W. A., Goode, P. R., Di Mauro, M. P., et al. 1998, ApJ, 509, 456

Elsworth, Y., Howe, R., Isaak, G. R., et al. 1990, Nature, 345, 322

Elsworth, Y., Howe, R., Isaak, G. R., et al. 1994, ApJ, 434, 801

Giles, P. M., Duvall Jr., T. L., Scherrer, P. H., et al. 1997, Nature, 390, 52

Howard, R., \& LaBonte, B. J. 1980, ApJ, 239, L33

Howe, R., Komm, R., \& Hill, F. 1999, ApJ, 524, 1084

Howe, R., Christensen-Dalsgaard, J., Hill, F., et al. 2000a, ApJL, 533, 163

Howe, R., Christensen-Dalsgaard, J., Hill, F., et al. 2000b, Science, 287, 2456

Howe, R., Komm, R., \& Hill, F. 2000c, Sol. Phys., 192, 427

Jain, R., \& Roberts, B. 1993, ApJ, 414, 898

Jain, R., \& Roberts, B. 1994, Sol. Phys., 152, 261

Komm, R., Howard, R. F., \& Harvey, J. W. 1993, Sol. Phys., 147, 207

Komm, R., Howe, R., \& Hill, F. 2000a, ApJ, 531, 1094

Komm, R., Howe, R., \& Hill, F. 2000b, ApJ, 543, 472

Libbrecht, K. G., \& Woodard, M. F. 1990, Nature, 345, 779

Murawski, K., \& Roberts, B. 1993, A\&A, 272, 595

Pintér, B., \& Goossens, M. 1999, A\&A, 347, 321

Pintér, B., Erdélyi, R., New, R., \& Goossens, M. 2001, in Helioand Astero-seismology at the Dawn of the Millennium, ed. A. Wilson, ESA SP-464, 227

Rhodes, E. J., Jr. Cacciani, A., Korzennik, S. G., \& Ulrich, R. K. 1993, ApJ, 406, 714

Roth, M., \& Stix, M. 1999, A\&A, 351, 1133

Toomre, J., Christensen-Dalsgaard, J., Howe, R., et al. 2000, Sol. Phys., 192, 437 